\title{
Temperamento, Prematuridade e Comportamento Interativo Mãe-Criança
}

\author{
Temperament, Prematurity and Mother-Child Interactive Behavior \\ Rafaela Guilherme Monte Cassiano* \& Maria Beatriz Martins Linhares \\ Universidade de São Paulo, Ribeirão Preto, SP, Brasil
}

\begin{abstract}
Resumo
Este estudo teve por objetivo elaborar e testar um modelo combinado de avaliação do temperamento de crianças, diferenciadas pela idade gestacional, e do comportamento em interação com suas mães. A amostra incluiu 10 crianças, sendo cinco nascidas a termo e cinco nascidas pré-termo, com idade entre 18 a 36 meses, e suas respectivas mães. A coleta de dados foi realizada em seus domicílios, onde foi aplicado com a mãe o The Early Childhood Behavior Questionnaire para avaliação do temperamento das crianças e realizada uma observação sistemática das interações mãe-criança em situação lúdica-livre. A análise de dados da interação focalizou o tipo de inicio dos episódios de contato, dinâmica das atividades, responsividade, sincronia, intrusividade, raiva ou frustração e oposicionismo. Os resultados mostraram que o temperamento das crianças nascidas a termo e pré-termo diferenciou-se quanto à expressão do Controle com Esforço e Extroversão. Os intercâmbios iniciados pela mãe foram os mais frequentes nas interações de ambos os grupos. Houve maior intrusividade materna e raiva e oposicionismo das crianças nascidas prematuras em comparação às nascidas a termo. O modelo de avaliação foi válido para identificar os padrões de comportamento das crianças com desenvolvimento típico e com prematuridade em interação com suas mães.

Palavras-chave: Temperamento, interação mãe criança, nascimento prematuro.
\end{abstract}

\begin{abstract}
The aim of this study was to develop and evaluate a combined model of temperament assessment of children, differentiated by gestational age, and behavior in the interaction with their mothers. The sample included 10 children, five born full term and five born preterm, aged 18 to 36 months, and their mothers. Data collection was performed in their homes, through an interview with mothers in order to assess their children's temperament using The Early Childhood Behavior Questionnaire. The study also carried out a systematic observation of the mother-child interactions in a free-play situation. The data analysis of the interaction focused on the type of beginning of contact episodes, dynamics of activities, responsiveness, synchronicity, intrusiveness, anger/frustration and opposition. Results showed that the temperament of children born full term and preterm differed for the expression of Effort Control and Extroversion. Exchanges started by mothers were the most frequent ones in the interactions of both groups. There were more intrusive mothers and children showed more anger and opposition in dyads of children born preterm compared to dyads of children born full term. The assessment model was useful in order to identify the behavior profiles of children with typical development as well as prematurity in interactions with their mothers.

Keywords: Temperament, mother-child interactions, premature birth.
\end{abstract}

A interação mãe-criança pode ser influenciada tanto por características da criança quanto por características maternas (Sameroff, 2009). Com relação às características das crianças destacam-se os fatores de risco biológico como a prematuridade (Poehlmann et al., 2011), malformação ao nascimento (Blacher, Baker, \& Kaladjian, 2013) e infecções congênitas (Swanson \& Schleiss, 2013), além

\footnotetext{
* Endereço para correspondência: Universidade de São Paulo, Faculdade de Medicina de Ribeirão Preto, Avenida Tenente Catão Roxo, 2650, Prédio da Saúde Mental, sala 53, Ribeirão Preto, SP, Brasil 14051-140. E-mail: rafaelagme@yahoo.com.br elinhares@fmrp.usp.br Processo $n^{\circ}$ 2010/12905-0, Fundação de Amparo à Pesquisa do Estado de São Paulo (FAPESP).
}

de fatores psicológicos, como o temperamento (Kim \& Kochanska, 2012) e comportamento (Lipscomb et al., 2012). As características maternas, por sua vez, que influenciam essa interação são a responsividade, que se refere ao modo como o adulto altera seu comportamento e o ambiente físico em reposta às demandas da criança (Gartstein, Crawford, \& Robertson, 2008; Kim \& Kochanska, 2012), intrusividade, relativa à falta de respeito à autonomia da criança (Adam, Gunnar, \& Tanaka, 2004; Taylor, Eisenberg, Spinrad, \& Widaman, 2013), sincronia, definida como uma interação mutuamente regulada, harmoniosa e recíproca (Feldman, 2007; Gartstein et al., 2008) e depressão (Feng et al., 2008). Além das características da díade, as variáveis contextuais, como a condição socio- 
econômica, atuam influenciando a qualidade da interação (Potharst et al., 2012).

Diante da importância da interação mãe-criança e o crescente aumento da sobrevivência de crianças nascidas prematuras (com idade gestacional menor do que 37 semanas), as interações têm sido investigadas em amostras de crianças com o risco biológico da prematuridade. As mães das crianças nascidas prematuras são caracterizadas por oferecerem menos suporte ao desenvolvimento de comportamentos autônomos nas crianças (Feldman, 2007; Potharst et al., 2012) e mais intrusivas do que as mães de crianças nascidas a termo (Poehlmann et al., 2011; Potharst et al., 2012).

Por outro lado, os bebês prematuros possuem menores capacidades autorregulatórias do que os nascidos a termo (Feldman, 2007, 2009). As crianças nascidas prematuras são caracterizadas por serem menos responsivas, menos atentas (Feldman, 2007, 2009), mais agitadas (Klein, Putnam, \& Linhares, 2009), e com menores quantidades de expressões faciais e comportamentos de busca do que as nascidas a termo (Feldman, 2007).

As dificuldades na autorregulação das crianças nascidas pré-termo afetam a expressão do seu temperamento (Klein et al., 2009). Com relação ao temperamento, a abordagem de Mary Rothbart, mais utilizada atualmente para o seu estudo (Klein \& Linhares, 2010), define este como as diferenças individuais com base constitucional na reatividade e autorregulação, nos domínios do afeto, atividade e atenção. $\mathrm{O}$ termo constitucional refere-se às bases relativamente biológicas do temperamento, influenciadas o tempo todo pela hereditariedade, maturação e experiência. A reatividade diz respeito às características da responsividade individual a mudanças de estimulação externa ou interna apresentada em diversos níveis. A autorregulação, por sua vez, consiste nos mecanismos usados pelo indivíduo para controlar suas reações emocionais e comportamentais frente a fontes de estimulação positiva ou negativa (Rothbart, 2004).

Destaca-se a importância de avaliar a relação entre temperamento e interação mãe-criança em amostras com o desenvolvimento típico e com a história de prematuridade. Recentemente, estudos de revisão indicam que existem poucos estudos sobre temperamento de prematuros, sendo que estes são mais centrados em avaliações por meio de relatos e não por observações sistemáticas (Cosentino-Rocha \& Linhares, 2013; Klein \& Linhares, 2010; Linhares, Dualibe, \& Cassiano, 2013). O objetivo do presente estudo foi elaborar e testar um modelo combinado de avaliação do temperamento de crianças, diferenciadas pela idade gestacional, e do comportamento no contexto interativo com a mãe, na fase de 18 a 36 meses de idade.

\section{Método}

\section{Participantes}

A amostra do estudo foi composta por 10 crianças com idade entre 18 a 36 meses, de ambos os sexos, e suas respectivas mães, sendo: cinco nascidas a termo, com idade gestacional maior do que 37 semanas (Grupo AT) e cinco nascidas pré-termo, com idade gestacional menor do que 37 semanas (Grupo PT). Os critérios de inclusão do estudo foram os seguintes: crianças entre 18 a 36 meses, filhos biológicos, mães como cuidadoras principais da criança e famílias de nível socioeconômico médio ou alto. Os critérios de exclusão foram: mães e crianças com deficiência cognitiva ou sob uso de medicações que pudessem alterar seu nível de consciência, mães com história de problemas de saúde mental, avaliado pela Entrevista Clínica Estruturada para o DSM-III-R Versão Não-Paciente (SCID-NP; Spitzer, Williams, Gibbon, \& First, 1989), e famílias de nível socioeconômico baixo, avaliado pelo Critério de Classificação Econômica da Associação Brasileira de Empresas e Pesquisa (Associação Brasileira de Empresas de Pesquisa [ABEP], 2012). A fonte de recrutamento foi realizada por meio de convites pessoais. O estudo foi aprovado pelo Comitê de Ética em Pesquisa da Faculdade de Filosofia Ciências e Letras de Ribeirão Preto, Universidade de São Paulo. As mães assinaram um termo de consentimento livre e esclarecido.

\section{Instrumentos e Medidas}

\section{Na avaliação do temperamento:}

The Early Childhood Behavior Questionnaire (ECBQ; Putnam, Gartstein, \& Rothbart, 2006), versão brasileira (Klein et al., 2009). O instrumento é composto por 201 itens que mensuram 18 dimensões de temperamento em crianças de 18 a 36 meses. As dimensões do temperamento estão organizadas em três grandes fatores, obtidos pelas médias dos escores de suas respectivas dimensões, a saber: (a) Extroversão: Nível de Atividade, Prazer de Alta Intensidade, Impulsividade, Antecipação Positiva e Sociabilidade; (b) Afeto Negativo: Frustração, Desconforto, Medo, Tristeza, Timidez, Ativação Motora, Sensibilidade Perceptual e do escore invertido da Capacidade de se Acalmar; (c) Controle com Esforço: Focalização da Atenção, Controle Inibitório, Prazer de Baixa Intensidade, Aconchego e Transferência da Atenção. O ECBQ é um instrumento respondido pela mãe/cuidador da criança, o qual deve indicar a frequência com que a criança apresentou determinada reação em contextos específicos, nas últimas duas semanas, atribuindo um valor em uma escala de Likert, que varia de 1 (nunca) a 7 (sempre). Putnam et al. (2006) demonstrou que a consistência interna das escalas variavam de 0,57 to 0,90 (média $\alpha=0,81$ ) e o acordo entre respondentes variando de 0,09 a 0,57 (média do $r=0,39$ ). Com relação à consistência interna das escalas do ECBQ traduzido para a Língua Portuguesa (Brasil), Klein et al. (2009) encontraram em seus dados uma variação de 0,43 a 0,88 (média $\alpha=0,72$ ).

\section{Procedimento}

Coleta de Dados. A coleta de dados ocorreu no período de maio de 2011 a fevereiro de 2012. Inicialmente foi 
agendada uma visita domiciliar, na qual foi administrada a SCID-NP (Spitzer et al., 1989) e a ABEP (2012), por meio de entrevista, a fim de verificar se os participantes atendiam aos critérios de inclusão, sendo que não houve exclusão de participantes. Após essa certificação, foi entregue à mãe o questionário ECBQ para ela responder de forma autoadministrada e entregar na próxima visita. A pesquisadora leu as instruções do questionário, esclareceu dúvidas e agendou uma segunda visita domiciliar. Durante a segunda visita, foi realizada a observação sistemática em situação lúdica-livre da interação mãe-criança, a qual foi filmada. Essa observação foi realizada no espaço da casa e com os brinquedos escolhidos pela mãe. Esta foi convidada a realizar uma brincadeira com seu filho, como usualmente brincam. As crianças foram esclarecidas sobre a presença da pesquisadora. A duração da observação foi estabelecida em 15 minutos, entretanto, se as díades estivessem executando uma atividade ao atingir este tempo, seria esperado o final da atividade para encerrar a observação. Caso houvesse alguma intercorrência não controlada durante a filmagem, esta deveria ser interrompida e retomada até atingir os 15 minutos de duração.

Preparação dos Dados. Os sistemas de análise utilizados para avaliar a interação estão descritos a seguir:

Sistema de Categorias de Análise de Episódios de Contato Mãe-criança (Linhares, Martins, \& Klein, 2004; Martins, 2001). Compreende as seguintes categorias de episódios de contato: (a) Intercâmbio iniciado pela mãe, mãe procura estabelecer comunicação com a criança e esta responde à comunicação; entende-se por comunicação qualquer verbalização ou atividade motora; (b) Intercâmbio iniciado pela criança, criança procura estabelecer comunicação com a mãe e esta responde à comunicação; (c) Iniciativa da mãe de intercâmbio, mãe procura estabelecer comunicação com a criança e esta não responde; (d) Iniciativa da criança de intercâmbio, criança procura estabelecer comunicação com a mãe e esta não responde; (e) Observação da criança pela mãe, mãe direciona o olhar para a criança, observando-a por tempo mínimo de cinco segundos; (f) Observação da mãe pela criança, criança direciona olhar para a mãe, observando-a por tempo mínimo de cinco segundos; (g) Ausência de interação/ Atividade Paralela, mãe e criança sem interação verbal ou motora por tempo mínimo de cinco segundos, podendo ou não estar desenvolvendo atividades paralelas de forma independente. Esse sistema foi aplicado, originalmente, em uma amostra de 15 crianças nascidas a termo e 15 crianças nascidas prematuras, com idade média de seis anos, durante uma interação lúdica com suas mães realizada em laboratório. Os índices de concordâncias obtidos entre dois observadores independentes na aplicação desse sistema foram de 95\% (Martins, 2001) e 91\% (Klein, 2005).

Sistema de Dinâmica da Díade Mãe-criança (Klein, 2005). Avalia a dinâmica da atividade desenvolvida na interação após o episódio de contato, sem considerar os materiais utilizados. As categorias desse sistema são: (a) Atividade compartilhada pela díade mãe-criança, mãe e criança realizam em conjunto ações sincronizadas em um mesmo intervalo de tempo e sincronizadas com a natureza da tarefa; (b) Atividade realizada pela criança com participação parcial da mãe, criança realiza ações vinculadas a uma atividade e a mãe a observa e tem participação verbal e/ou motora na atividade parcialmente, de forma sincronizada e sintonizada; (c) Atividade realizada pela criança de forma independente da mãe, criança realiza ações vinculadas a uma atividade enquanto a mãe não se envolve em nenhuma atividade realizada pela criança; (d) Atividade realizada pela mãe de forma independente da criança, mãe realiza ações vinculadas a uma atividade enquanto a criança não se envolve em nenhuma atividade realizada pela mãe; (e) Atividade paralela da Díade Mãe-Criança, mãe e criança realizam, independentemente uma da outra e em um mesmo intervalo de tempo, ações vinculadas a atividades que podem ser similares ou diferentes. A partir da aplicação desse sistema no presente estudo, foi constatada a necessidade de acrescentar a categoria Atividade realizada pela mãe com participação parcial da criança, definida por quando a mãe realiza ações vinculadas a uma atividade e a criança a observa e tem participação verbal e/ou motora na atividade parcialmente, de forma sincronizada e sintonizada. O índice de concordância obtido na aplicação original desse sistema por dois juízes independentes foi de $85 \%$ (Klein, 2005).

Escala de Gartstein et al. (2008). Traduzida e adaptada para este estudo com autorização da autora. Essa escala possui escores que variam de um a sete em uma escala Likert, a qual avalia a Responsividade/Sensibilidade materna (escore qualitativamente positivo $=7$ ) e Reciprocidade/Sincronia (escore qualitativamente positivo $=7$ ). A confiabilidade interobservador obtida na aplicação original foi de $82 \%$ (Gartstein et al., 2008).

Sistema de Análise do Comportamento Interativo Materno (Adam et al., 2004). Traduzido e adaptado para a presente pesquisa, avaliando a Intrusividade materna. A partir desse sistema foram criadas categorias semelhantes para avaliar o comportamento interativo da criança, as quais avaliaram o Oposicionismo e Raiva ou Frustração da criança. Essas categorias foram avaliadas em uma escala de zero a dois, na qual zero corresponde a nenhuma ou baixa quantidade de comportamento, um significa pequena quantidade e dois corresponde à grande quantidade do comportamento.

Primeiramente, foram aplicados o Sistema de Categorias de Análise de Episódios de Contato e o Sistema de Dinâmica da Díade Mãe-criança, por meio do programa The Observer XT (versão 9.0; Noldus, EUA), utilizando-se a unidade de tempo em segundos. Ao final, foi obtido um coeficiente de confiabilidade intraobservador (Kappa) de 0,80 . A categorização dos dados por meio desses sistemas foi realizada pela pesquisadora e, posteriormente, reavaliada por outra pesquisadora, ambas treinadas e experientes em observação sistemática do comportamento e desenvolvimento infantil. Após essas categorizações, os dados observacionais foram avaliados pelas escalas e 
Cassiano, R. G. M. \& Linhares, M. B. M. (2015). Temperamento, Prematuridade e Comportamento Interativo Mãe-Criança.

os escores finais computados foram obtidos por meio de consenso entre as duas pesquisadoras. Essa análise foi realizada intencionalmente após as demais, pois exigiam que as pesquisadoras apresentassem familiaridade com a dinâmica das díades.

\section{Análise dos Dados}

Os dados foram submetidos à análise de estatística descritiva para cada grupo de crianças, em termos de: (a) média, desvio-padrão, no caso das variáveis numéricas contínuas; (b) frequência, porcentagem e duração no caso das variáveis categóricas. Os dados foram processados pelo Statistical Package for Social Sciences (SPSS, versão 19.0, IL, Chicago, USA), exceto os dados dos sistemas de análise das observações os quais foram analisados por meio do programa The Observer XT.

\section{Resultados}

\section{Características da Amostra de Estudo}

A Tabela 1 mostra que as crianças do Grupo AT tinham em média 3.121 gramas ao nascimento, idade gestacional em torno de 39 semanas e a idade cronológica média de 25 meses. Por outro lado, as crianças do Grupo PT pesavam, em média, 1.778 gramas ao nascimento, com idade gestacional média de 32 semanas e idade média de 22 meses. Ambos os grupos foram compostos por três meninas e dois meninos.

As mães e os pais do Grupo AT eram adultos jovens ao nascimento das crianças, com idade média, respectivamente, de 29 e 30 anos. O nível de escolaridade materno situou-se entre os níveis médio e superior, com predomínio deste último. Por outro lado, todos os pais desse grupo possuíam o Ensino Médio, eram empregados e casados. O nível socioeconômico dessas famílias era B1 e B2, de acordo com a ABEP (2012). Essas classificações estão associadas a uma renda mensal, respectivamente, de $\mathrm{R} \$$ $4.418,00$ e $\mathrm{R} \$ 2.565,00$.

No Grupo PT, por sua vez, as mães e os pais também eram adultos jovens, com idade média, respectivamente, de 30 e 32 anos ao nascimento das crianças. O nível de escolaridade materno predominante foi o superior completo e apenas uma mãe era do lar. Todos os pais possuíam o Ensino Médio, eram casados e empregados. As classificações socioeconômicas encontradas, segundo a ABEP (2012), foram A2, B1, B2 e C1, as quais possuem renda média, respectivamente, de $\mathrm{R} \$ 8.418,00, \mathrm{R} \$ 4.418,00, \mathrm{R} \$$ $2.565,00$ e $\mathrm{R} \$ 1.541,00$.

Tabela 1

Características das Crianças do Grupo de Crianças Nascidas A Termo (Grupo AT) e Pré-Termo (Grupo PT)

\begin{tabular}{lcccc} 
Características das crianças & $\begin{array}{c}\text { Peso ao nascimento } \\
\text { (gramas) Média } \\
(D P)\end{array}$ & $\begin{array}{c}\text { Idade gestacional } \\
\text { (semanas) Média } \\
(D P)\end{array}$ & $\begin{array}{c}\text { Idade da criança } \\
\text { (meses) Média } \\
(D P)\end{array}$ & $\begin{array}{c}\text { Sexo } \\
\mathrm{F} / \mathrm{M} \\
f(\%)\end{array}$ \\
\hline Grupo AT & $3.121( \pm 383)$ & $39( \pm 1,34)$ & $25( \pm 5,55)$ & $3(60) / 2(40)$ \\
1AT & 2.870 & 41 & 19 & $\mathrm{~F}$ \\
2AT & 2.750 & 38 & 29 & $\mathrm{~F}$ \\
3AT & 3.450 & 38 & 23 & $\mathrm{M}$ \\
4AT & 2.925 & 38 & 33 & $\mathrm{~F}$ \\
5AT & 3.610 & 38 & 23 & $\mathrm{M}$ \\
Grupo PT & $1.778( \pm 443)$ & $32( \pm 2,12)$ & $22( \pm 2,12)$ & $3(60) / 2(40)$ \\
1PT & 1.930 & 35 & 20 & $\mathrm{~F}$ \\
2PT & 1.500 & 30 & 23 & $\mathrm{~F}$ \\
3PT & 1.735 & 32 & 20 & $\mathrm{M}$ \\
4 PT & 1.285 & 30 & 22 & $\mathrm{M}$ \\
5PT & 2.440 & 33 & 25 & $\mathrm{~F}$
\end{tabular}

Nota $. n=10$. Grupo AT = crianças nascidas a termo, com idade gestacional maior do que 37 semanas; Grupo PT $=$ crianças nascidas pré-termo, com idade gestacional menor do que 37 semanas; $D P=$ desvio padrão; $f=$ frequência; $\%=$ porcentagem; $\mathrm{F}=$ feminino; $\mathrm{M}=$ masculino.

Características do Temperamento das Crianças Nascidas A Termo e Pré-Termo

Verifica-se na Tabela 2 que os escores dos fatores do temperamento das crianças nascidas a termo apresentaram a seguinte ordem crescente: Afeto Negativo, Extroversão e Controle com Esforço. Por outro lado, as crianças nascidas pré-termo apresentaram a ordem crescente: Afeto Negativo, Controle com Esforço e Extroversão. 
Tabela 2

Indicadores do Temperamento das Crianças (Fatores) Avaliados pelo ECBQ, na Faixa de 18 a 36 Meses de Idade, no Grupo de Crianças Nascidas A Termo (Grupo AT) e Pré-Termo (Grupo PT)

\begin{tabular}{lccc}
\hline $\begin{array}{c}\text { Temperamento } \\
\text { Fatores (Escores) }\end{array}$ & Afeto Negativo & Extroversão & Controle com Esforço \\
\hline Grupo AT - Média (DP) & $3,38( \pm 0,38)$ & $5,00( \pm 0,52)$ & $5,07( \pm 0,49)$ \\
1 AT & 3,4 & 4,9 & 5,1 \\
$2 \mathrm{AT}$ & 3,0 & 4,2 & 5,8 \\
3AT & 3,6 & 5,2 & 4,5 \\
4AT & 3,9 & 5,6 & 5,0 \\
$5 \mathrm{AT}$ & 3,0 & 5,1 & 5,0 \\
Grupo PT - Média $(D P)$ & $3,12( \pm 0,26)$ & $4,94( \pm 0,51)$ & $4,65( \pm 0,69)$ \\
1PT & 3,4 & 4,6 & 4,5 \\
2PT & 3,3 & 5,6 & 3,9 \\
3PT & 2,9 & 4,4 & 5,2 \\
4 PT & 2,8 & 4,9 & 5,5 \\
5PT & 3,2 & 5,3 & 4,1 \\
\hline
\end{tabular}

Nota $. n=10$. Grupo AT = crianças nascidas a termo, com idade gestacional maior do que 37 semanas; Grupo PT $=$ crianças nascidas pré-termo, com idade gestacional menor do que 37 semanas; $D P=$ Desvio Padrão; escores variam de 1 a 7.

Tabela 3

Tipos de Episódios de Contato da Interação Mãe-Criança no Grupo de Crianças Nascidas A Termo (Grupo AT) e Pré-Termo (Grupo PT)

\begin{tabular}{|c|c|c|c|c|c|c|c|}
\hline $\begin{array}{l}\text { Episódios } \\
\text { de contato }\end{array}$ & $\begin{array}{l}\mathrm{M} \leftrightarrow \mathrm{C} \\
f(\%)\end{array}$ & $\begin{array}{c}\mathrm{C} \leftrightarrow \mathrm{M} \\
f(\%)\end{array}$ & $\begin{array}{l}\mathrm{M} \rightarrow \mathrm{C} \\
f(\%)\end{array}$ & $\begin{array}{c}\mathrm{C} \rightarrow \mathrm{M} \\
f(\%)\end{array}$ & $\begin{array}{c}\mathrm{M}^{\circ} \rightarrow \mathrm{C} \\
f(\%)\end{array}$ & $\begin{array}{c}\mathrm{C}^{\circ} \rightarrow \mathrm{M} \\
f(\%)\end{array}$ & $\begin{array}{l}\mathrm{M} / \mathrm{C} \\
f(\%)\end{array}$ \\
\hline \multicolumn{8}{|l|}{ Grupo AT } \\
\hline $1 \mathrm{AT}$ & $15(39)$ & $3(8)$ & $4(11)$ & 0 & $14(37)$ & 0 & $2(5)$ \\
\hline $2 \mathrm{AT}$ & $4(67)$ & $1(16,5)$ & $1(16,5)$ & 0 & 0 & 0 & 0 \\
\hline $3 \mathrm{AT}$ & $18(69)$ & $1(4)$ & $3(11)$ & $1(4)$ & $1(4)$ & $1(4)$ & $1(4)$ \\
\hline $4 \mathrm{AT}$ & $14(50)$ & $4(14)$ & $5(18)$ & $1(4)$ & $4(14)$ & 0 & 0 \\
\hline $5 \mathrm{AT}$ & $13(73)$ & $1(5)$ & 0 & 0 & $1(5)$ & $1(5)$ & $2(12)$ \\
\hline \multicolumn{8}{|l|}{ Grupo PT } \\
\hline $1 \mathrm{PT}$ & $10(63)$ & $4(25)$ & $2(12)$ & 0 & 0 & 0 & 0 \\
\hline $2 \mathrm{PT}$ & $10(50)$ & $7(35)$ & $2(10)$ & $1(5)$ & 0 & 0 & 0 \\
\hline $3 \mathrm{PT}$ & $7(26)$ & $3(11)$ & $15(55)$ & $1(4)$ & $1(4)$ & 0 & 0 \\
\hline $4 \mathrm{PT}$ & $12(31)$ & $4(11)$ & $11(29)$ & $2(5)$ & $8(21)$ & 0 & $1(3)$ \\
\hline $5 \mathrm{PT}$ & $9(60)$ & $3(20)$ & $3(20)$ & 0 & 0 & 0 & 0 \\
\hline
\end{tabular}

Nota. $n=10$. Grupo AT = crianças nascidas a termo; Grupo PT= crianças nascidas pré-termo; $\mathrm{M} \leftrightarrow \mathrm{C}=$ Intercâmbio iniciado pela mãe; $C \leftrightarrow M=$ Intercâmbio iniciado pela criança; $M \rightarrow C=$ Iniciativa da mãe de intercâmbio; $C \rightarrow M=$ Iniciativa da criança de intercâmbio; $\mathrm{M} \rightarrow \mathrm{C}=$ Observação da criança pela mãe; $\mathrm{C} \rightarrow \mathrm{M}=$ Observação da mãe pela criança; $\mathrm{M} / \mathrm{C}=\mathrm{Ausência} \mathrm{de} \mathrm{interação/}$ Atividade Paralela; $f=$ frequência; $\%=$ porcentagem. 
Características das Interações Mãe-Criança

De acordo com a Tabela 3, pode-se observar que em todas as díades do Grupo AT houve maior porcentagem dos intercâmbios iniciados pelas mães em comparação aos demais. A díade 1AT destaca-se pela elevada porcentagem dos episódios de observação da criança pela mãe em comparação com as outras díades.

No Grupo PT, por sua vez, a maior porcentagem dos episódios de contato foi de intercâmbios iniciados pela mãe na maioria das díades, exceto na díade 3PT. Destacam-se os altos valores obtidos pelas mães das díades 3PT e 4PT nas iniciativas de intercâmbio sem resposta da criança em relação às outras mães. Além disso, ressalta-se o valor obtido pela díade $4 \mathrm{PT}$ nos episódios de observação da criança pela mãe em relação às demais díades.

A Tabela 4 mostra que no Grupo AT a atividade compartilhada obteve maior porcentagem nas díades $2 \mathrm{AT}$ e
4AT em relação às demais dinâmicas. A atividade realizada pela criança com participação parcial da mãe apresentou maior porcentagem na díade $5 \mathrm{AT}$ em relação às outras atividades dessa díade. A díade 1AT obteve o predomínio da atividade realizada pela criança com participação parcial da mãe e da atividade realizada pela criança de forma independente da mãe, ambas com os valores idênticos de porcentagem. Por outro lado, a díade 3AT apresentou maior porcentagem da atividade realizada pela mãe com participação parcial da criança em relação às demais.

No Grupo PT, por sua vez, três díades (3PT, 4PT e 5PT) apresentaram maior porcentagem nas atividades realizadas pelas crianças com participação parcial da mãe, em comparação às outras dinâmicas. Por outro lado, as díades $1 \mathrm{PT}$ e 2PT obtiveram maior porcentagem nas atividades compartilhadas, em relação às outras dinâmicas e às demais díades.

Tabela 4

Frequência e Porcentagem das Dinâmicas das Atividades Desenvolvidas na Interação Mãe-Criança no Grupo de Crianças Nascidas A Termo (Grupo AT) e Pré-Termo (Grupo PT)

\begin{tabular}{lcccccc}
\hline $\begin{array}{l}\text { Dinâmica das } \\
\text { Atividades }\end{array}$ & $\begin{array}{l}\text { COMP } \\
f(\%)\end{array}$ & $\begin{array}{c}\text { CPPM } \\
f(\%)\end{array}$ & $\begin{array}{c}\text { MPPC } \\
f(\%)\end{array}$ & $\begin{array}{c}\text { CIM } \\
f(\%)\end{array}$ & $\begin{array}{c}\text { MIC } \\
f(\%)\end{array}$ & $\begin{array}{c}\text { AP } \\
\text { Grupo AT }\end{array}$ \\
$\begin{array}{llccc}\text { 1AT } \\
\text { 2AT }\end{array}$ & $7(19)$ & $13(35)$ & $2(5,5)$ & $13(35)$ & 0 & $2(5,5)$ \\
3AT & $4(80)$ & $1(20)$ & 0 & 0 & 0 & 0 \\
4 AT & $6(21)$ & $6(21)$ & $10(34)$ & $4(14)$ & $2(7)$ & $1(3)$ \\
5 AT & $8(40)$ & $7(35)$ & $3(15)$ & $2(10)$ & 0 & 0 \\
Grupo PT & $8(37)$ & $9(40)$ & $2(9)$ & 0 & $1(5)$ & $2(9)$ \\
1 PT & & & & & & \\
2PT & $14(66)$ & $6(29)$ & $1(5)$ & 0 & 0 & 0 \\
3PT & $16(80)$ & $2(10)$ & $1(5)$ & 0 & 0 & $1(5)$ \\
4 PT & $3(17)$ & $9(50)$ & $2(11)$ & $2(11)$ & 0 & $2(11)$ \\
5 PT & $7(23)$ & $12(39)$ & $4(13)$ & $6(19)$ & 0 & $2(6)$ \\
\hline
\end{tabular}

Nota $. n=10$. Grupo AT = crianças nascidas a termo; Grupo PT = crianças nascidas prematuras; COMP = Atividade compartilhada pela díade mãe-criança; $\mathrm{CPPM}=$ Atividade realizada pela criança com participação parcial da mãe; $\mathrm{MPPC}=$ Atividade realizada pela mãe com participação parcial da criança; $\mathrm{CIM}=$ Atividade realizada pela criança de forma independente da mãe; MIC = Atividade realizada pela mãe de forma independente da criança; $\mathrm{AP}=$ Atividade paralela da díade mãe-criança; $f=$ frequência; $\%=$ porcentagem .

Na Tabela 5, as mães do Grupo AT, em média, apresentaram alto ou moderado escore de responsividade e sincronia. As díades $2 \mathrm{AT}$ e $4 \mathrm{AT}$ possuem as mães mais responsivas e as interações com maior sincronia. Por outro lado, a díade 1AT apresentou a mãe menos sensível e a menor sincronia. A intrusividade materna foi exibida apenas pela mãe da díade $5 \mathrm{AT}$, sendo que nenhuma criança expressou oposicionismo e raiva ou frustração na interação.
No Grupo PT, as díades apresentaram ampla variação quanto aos escores obtidos. As mães das díades 1PT e 2PT foram as mais responsivas e mais sincrônicas em relação às outras díades. No entanto, as mães das díades 3PT e 4PT foram as menos responsivas e apresentaram a interação com menor sincronia em relação ás demais. Além disso, essas mães apresentaram intrusividade e suas crianças apresentaram oposicionismo e raiva na interação. 
Psicologia: Reflexão e Crítica, 28(2), 416-424.

Tabela 5

Escores das Características da Interação Mãe-Criança do Grupo de Crianças Nascidas A Termo (Grupo AT) e Pré-Termo (Grupo PT)

\begin{tabular}{|c|c|c|c|c|c|}
\hline $\begin{array}{l}\text { Características } \\
\text { da interação-Escores }\end{array}$ & $\begin{array}{c}\text { Responsividade/ } \\
\text { Sensibilidade Materna }^{1}\end{array}$ & $\begin{array}{l}\text { Reciprocidade/ } \\
\text { Sincronia }^{1}\end{array}$ & $\begin{array}{l}\text { Intrusividade } \\
\text { materna }^{2}\end{array}$ & $\begin{array}{c}\text { Criança } \\
\text { opositora }^{2}\end{array}$ & $\begin{array}{c}\text { Raiva/Frustração } \\
\text { da criança }{ }^{2}\end{array}$ \\
\hline $\begin{array}{l}\text { Grupo AT } \\
\text { Média }(D P)\end{array}$ & $5,2( \pm 1,79)$ & $5( \pm 2,12)$ & $0,2( \pm 0,45)$ & 0 & 0 \\
\hline $1 \mathrm{AT}$ & 3 & 2 & 0 & 0 & 0 \\
\hline $2 \mathrm{AT}$ & 7 & 7 & 0 & 0 & 0 \\
\hline $3 \mathrm{AT}$ & 4 & 4 & 0 & 0 & 0 \\
\hline $4 \mathrm{AT}$ & 7 & 7 & 0 & 0 & 0 \\
\hline $5 \mathrm{AT}$ & 5 & 5 & 1 & 0 & 0 \\
\hline \multicolumn{6}{|l|}{ Grupo PT } \\
\hline Média $(D P)$ & $4,8( \pm 2,28)$ & $5( \pm 2)$ & $0,6( \pm 0,89)$ & $0,6( \pm 0,89)$ & $0,6( \pm 0,89)$ \\
\hline $1 \mathrm{PT}$ & 7 & 7 & 0 & 0 & 0 \\
\hline $2 \mathrm{PT}$ & 7 & 7 & 0 & 0 & 0 \\
\hline $3 \mathrm{PT}$ & 2 & 3 & 2 & 2 & 2 \\
\hline $4 \mathrm{PT}$ & 3 & 3 & 1 & 1 & 1 \\
\hline $5 \mathrm{PT}$ & 5 & 5 & 0 & 0 & 0 \\
\hline
\end{tabular}

Nota $. n=10$. Grupo AT = crianças nascidas a termo, com idade gestacional maior do que 37 semanas; Grupo PT $=$ crianças nascidas pré-termo, com idade gestacional menor do que 37 semanas, $D P=$ desvio padrão; ${ }^{1}=$ escores variam de 1 a $7 ;^{2}=$ escores variam de 0 a 2 .

\section{Discussão}

O presente estudo examinou as características do temperamento infantil e a interação mãe-criança em situação lúdica-livre, em cinco crianças nascidas a termo e cinco crianças pré-termo. Quanto ao temperamento infantil, as crianças nascidas a termo apresentaram os maiores escores nos fatores na seguinte ordem crescente: Afeto Negativo, Extroversão e Controle com Esforço. Por outro lado, as crianças nascidas pré-termo apresentaram escores nos fatores na ordem crescente: Afeto Negativo, Controle com Esforço e Extroversão. Verifica-se, no entanto, que as médias da Extroversão e do Controle com Esforço foram muito próximas.

Essa disposição dos fatores do temperamento coincide com a etapa do desenvolvimento infantil da faixa etária estudada, de 18 a 36 meses, em que sistemas mais regulados estão se desenvolvendo. Aos dois anos de idade, a habilidade da atenção sustentada está desenvolvida, influenciando diretamente a expressão do fator Controle com Esforço. Além disso, a regulação emocional estabelecida durante os dois primeiros anos de idade interfere na diminuição do fator Afeto Negativo (Rothbart \& Bates, 2006). Destaca-se que o temperamento infantil pode atuar como um fator protetor ou de risco ao desenvolvimento infantil, podendo facilitar ou dificultar a interação mãe-criança (Kim \& Kochanska, 2012).

Quanto à situação de observação lúdica-livre do presente estudo houve um predomínio dos intercâmbios iniciados pelas mães em todas as díades do Grupo AT e em quatro das cinco díades do Grupo PT. Resultados semelhantes foram encontrados no estudo de Klein (2005) em interações lúdicas de mães com crianças nascidas a termo e pré-termo, aos seis anos de idade.

As dinâmicas das atividades identificadas no presente estudo refletem as semelhanças e as especificidades do comportamento das díades. Com relação ao Grupo AT, a atividade compartilhada pela mãe e criança apresentou maior porcentagem em duas díades (2AT e 4AT) em relação às demais dinâmicas. Por outro lado, no Grupo PT houve uma maior porcentagem das atividades realizadas pelas crianças com participação parcial da mãe (3PT, 4PT e 5PT). No entanto, duas díades (1PT e 2PT) obtiveram maior porcentagem das atividades compartilhadas em comparação às demais.

Na faixa etária de 18 a 36 meses é mais indicada a atividade compartilhada, devido a essa fase ser um marco para o desenvolvimento da autorregulação e necessitar de um comportamento materno mais ativo na interação. A mãe atua como uma correguladora externa aos comportamentos da criança, a fim de que esta internalize esses aspectos ao seu processo autorregulatório (Bernier, Whipple, \& Carlson, 2010). Destaca-se que a atividade compartilhada está intrinsecamente relacionada com o fenômeno da atenção conjunta descrito por Tomasello (2003) como a habilidade de coordenar a atenção entre um parceiro social e um objeto de interesse mútuo. Além disso, essa dinâmica pode atuar na zona de desenvolvimento proxi- 
mal, definida por Vygotsky (1930/1998) como a distância entre o nível de desenvolvimento real, determinado pela capacidade de resolver um problema sem ajuda, e o nível de desenvolvimento potencial, determinado por meio de resolução de um problema sob a orientação de um adulto ou em colaboração com outra pessoa mais capaz.

A categoria atividade compartilhada é semelhante à dinâmica descrita por Kochanska (2002) como orientação mutuamente responsiva, caracterizada por ser uma ligação mútua, cooperativa, com responsividade mútua e compartilhamento de afeto positivo. Essa dinâmica mostrou ser um fator protetor aos 25 meses de idade para as crianças que possuíam emocionalidade negativa aos sete meses de idade. Crianças com emocionalidade negativa foram menos autorreguladas quando elas estavam em interação mãe-criança com baixa orientação mutuamente responsiva, no entanto, eram mais autorreguladas quando em interações mutuamente responsivas (Kim \& Kochanska, 2012).

No presente estudo, os escores obtidos nas escalas de Gartstein et al. (2008) mostraram que a maioria das mães dos dois grupos apresentou alto ou moderado escore de responsividade/sensibilidade e reciprocidade/sincronia, o que caracteriza uma interação materna positiva. Os comportamentos maternos como responsividade e sincronia atuam como reguladores externos ao comportamento da criança, os quais fortalecem o seu processo de autorregulação (Bernier et al., 2010). Deve-se ressaltar que o temperamento infantil também pode ser influenciado pela qualidade dessa interação. Durante a interação mãe-criança, a responsividade materna atuou como preditora da dimensão do temperamento infantil Sensibilidade Perceptual aos 12 meses de idade (Gartstein et al., 2008). Além disso, o surgimento do fator do Controle com Esforço no temperamento da criança está intimamente relacionado com a sensibilidade e afetividade materna (Bridgett et al., 2011).

Nos resultados do Grupo PT, as mães das díades 3PT e 4PT exibiram intrusividade, enquanto as crianças apresentaram oposicionismo e raiva ou frustração, indicando dificuldades nos comportamentos interativos. A intrusividade materna no Grupo PT vai ao encontro dos resultados das pesquisas na área (Feldman, 2007; Poehlmann et al., 2011; Potharst et al., 2012). A intrusividade em mães de crianças prematuras é adicionada à condição de risco da prematuridade, constituindo-se em múltiplo risco ao desenvolvimento dessas crianças. Além disso, a intrusividade materna interage com o temperamento infantil. Crianças nascidas prematuras em famílias com baixo nível socioeconômico, cujas mães apresentaram raiva ou críticas durante a interação, apresentaram menor Controle com Esforço aos dois anos de idade, em comparação às crianças prematuras com mães com comportamentos mais positivos (Poehlmann et al., 2011).

De modo interessante, com relação à análise da interação das 10 díades, observa-se que as crianças que apresentaram raiva na interação são as que possuem mães com menores habilidades interativas e realizaram uma elevada porcentagem de iniciativas de intercâmbio sem resposta da criança. Por outro lado, as díades que apresentaram maior porcentagem de atividades compartilhadas correspondiam às mães com mais habilidades interativas.

A metodologia de análise da interação mãe-criança do presente estudo utilizou um conjunto de instrumentos que propiciaram uma análise detalhada das interações em ambiente natural. O tempo de observação estabelecido em 15 minutos se mostrou eficaz para caracterizar as interações, permitindo uma ampla análise dos dados. Além disso, esse modelo combinado de avaliação foi capaz de identificar diferenças nos padrões interativos entre mães e crianças com desenvolvimento típico ou com história da prematuridade.

A condição de risco da prematuridade, quando associada a poucas qualidades interativas maternas e pobres capacidades autorregulatórias da criança, resultou em uma interação com um pior desempenho em comparação às outras díades pré-termo e a termo. Como desdobramento dos achados recomenda-se o desenvolvimento de programas de intervenção que estimulem e ensinem mães de crianças em risco a atuarem como correguladoras do desenvolvimento infantil (Linhares, Gaspardo, \& Klein, 2012).

$\mathrm{O}$ presente estudo possui algumas limitações com relação ao número de participantes e não possuir participantes de nível socioeconômico baixo, o que ajudaria na generalização dos achados. Destaca-se que o número de participantes não permitiu análises estatísticas visando à comparação entre grupos. Como sugestão para futuras pesquisas, propõe-se a aplicação desse modelo de avaliação em programas de intervenção, a fim de avaliar as mudanças na qualidade da interação mãe-criança associadas ao temperamento infantil. Além disso, sugere-se que esse modelo inclua a avaliação do temperamento materno.

\section{Referências}

Adam, E. K., Gunnar, M. R., \& Tanaka, A. (2004). Adult attachment, parent emotion, and observed parenting behavior: Mediator and moderator models. Child Development, 75(1), 110-122. doi:10.1111/j.1467-8624.2004.00657.x

Associação Brasileira de Empresas de Pesquisa. (2012). Critério de classificação econômica Brasil. São Paulo, SP: Autor. Recuperado em 25 de março de 2012, de http://www.abep. org/novo/Content.aspx?ContentID $=301$

Bernier, A., Whipple, N., \& Carlson, S. M. (2010). From external regulation to self-regulation: Early parenting precursors of young children's executive functioning. Child Development, 81(1), 326-339. doi:10.1111/j.1467-8624.2009.01397.x

Blacher, J., Baker, B. L., \& Kaladjian, A. (2013). Syndrome specificity and mother-child interactions: Examining positive and negative parenting across contexts and time. Journal of Autism and Developmental Disorders, 43(4), 761-774. doi:10.1007/s10803-012-1605-x

Bridgett, D. J., Gartstein, M. A., Putnam, S. P., Lance, K. O., Iddins, E., Waits, R., ...Lee, L. (2011). Emerging effortful control in toddlerhood: The role of infant orienting/regulation, maternal effortful control, and maternal time spent in caregiving activities. Infant Behavior \& Development, 34(1), 189-199. doi:10.1016/j.infbeh.2010.12.008 
Cosentino-Rocha, L., \& Linhares, M. B. M. (2013). Temperamento de crianças e diferenças de gênero. Paidéia (Ribeirão Preto), 23(54), 63-72. doi:10.1590/1982-43272354201308

Feldman, R. (2007). Parent-infant synchrony and the construction of shared timing, physiological precursors, developmental outcomes, and risk conditions. Journal of Child Psychology and Psychiatry, 48(3), 329-354. doi:10.1111/j.14697610.2006.01701.x

Feldman, R. (2009). The development of regulatory functions from birth to 5 years: Insights from premature infants. Child Development, 80(2), 544-561. doi:10.1111/j.14678624.2009.01278.x

Feng, X., Shaw, D. S., Kovacs, M., Lane, T., O’Rourke, F. E., \& Alarcon, J. H. (2008). Emotion regulation in preschoolers: The roles of behavioral inhibition, maternal affective behavior, and maternal depression. Journal of Child Psychology and Psychiatry, 49(2), 132-141. doi:10.1111/j.14697610.2007.01828.x

Gartstein, M. A., Crawford, J., \& Robertson, C. R. (2008). Early markers of language and attention: Mutual contributions and the impact of parent-infant interactions. Child Psychiatry \& Human Development, 39(1), 9-26. doi:10.1007/s10578007-0067-4

Kim, S., \& Kochanska, G. (2012). Child temperament moderates effects of parent-child mutuality on self-regulation: A relationship based path for emotionally negative infants. Child Development, 83(4), 1275-1289. doi:10.1111/j.14678624.2012.01778.x

Klein, V. C. (2005). Interação entre mães e crianças pré-escolares nascidas pré-termo e com muito baixo peso em situação lúdica e de ensino (Dissertação de mestrado, Faculdade de Medicina de Ribeirão Preto, Universidade de São Paulo, Ribeirão Preto, SP, Brasil). Recuperada em http://pgsm.fmrp.usp.br/ wp-content/uploads/2011/11/Disserta $\%$ C3\%A7\%C3\%A3o Vivian.pdf_.pdf

Klein, V. C., \& Linhares, M. B. M. (2010). Temperamento e desenvolvimento: Revisão sistemática da literatura. Psicologia em Estudo, 15(4), 821-829. doi:10.1590/S141373722010000400018

Klein, V. C., Putnam, S. P., \& Linhares, M. B. L. (2009). Assessment of temperament in children: Translation of instruments to Portuguese (Brazil) language. Interamerican Journal of Psychology, 43(3), 552-557. Retrieved from http://pepsic. bvsalud.org/pdf/rip/v43n3/v43n3a15.pdf

Kochanska, G. (2002). Mutually responsive orientation between mothers and their young children: A context for the early development of conscience. Current Directions in Psychological Science, 11(191), 191-195. doi:10.1111/1467-8721.00198

Linhares, M. B. M., Dualibe, A. L., \& Cassiano, R. G. M. (2013). Temperamento de crianças na abordagem de Rothbart: Estudo de revisão sistemática. Psicologia em Estudo, 18(4), 633-645. doi:10.1590/S1413-73722013000400006

Linhares, M. B. M., Gaspardo, C. M., \& Klein, V. C. (2012). O impacto do nascimento pré-termo no desenvolvimento da criança e na família. In T. I. J. S. Riechi \& M. V. L. Moura-Ribeiro (Eds.), Desenvolvimento de crianças nascidas pré-termo (pp.47-70). Rio de Janeiro, RJ: Revinter.

Linhares, M. B. M., Martins, I. M. B., \& Klein, V. C. (2004). Mediação materna como processo de promoção e proteção do desenvolvimento da criança nascida prematura. In E. M. Maturano, M. B. M. Linhares, \& S. R. Loureiro (Eds.), Vulnerabilidade e proteção: Indicadores na trajetória de desenvolvimento do escolar (pp. 39-74). São Paulo, SP: Casa do Psicólogo.
Lipscomb, S. T., Leve, L. D., Shaw, D. S., Neiderhiser, J. M., Scaramella, L. V., Ge, X., ...Reiss, D. (2012). Negative emotionality and externalizing problems in toddlerhood: Overreactive parenting as a moderator of genetic influences. Development and Psychopathology, 24(1), 167-79. doi:10.1017/S0954579411000757

Martins, I. M. B. (2001). Crianças nascidas pré-termo e muito baixo peso, na fase pré-escolar: História de desenvolvimento, comportamento e mediação materna (Dissertação de mestrado não-publicada, Faculdade de Filosofia Ciências e Letras de Ribeirão Preto, Universidade de São Paulo, Ribeirão Preto, SP, Brasil).

Poehlmann, J., Schwichtenberg, A. J. M., Shlafer, R. J., Hahn, E., Bianchi, J. P., \& Warner, R. (2011). Emerging self-regulation in toddlers born preterm or low birth weight: Differential susceptibility to parenting? Development and Psychopathology, 23(1), 177-193. doi:10.1017/S0954579410000726

Potharst, E. V., Schuengel, C., Last, B. F., Wassenaer, A. G., Kok, J. H., \& Houtzager, B. A. (2012). Difference in mother-child interaction between preterm- and term-born preschoolers with and without disabilities. Acta Paediatrica, 101(6), 597-603. doi:10.1111/j.1651-2227.2012.02599.x

Putnam, S. P., Gartstein, M. A., \& Rothbart, M. K. (2006). Measurement of fine-grained aspects of toddler temperament: The early childhood behavior questionnaire. Infant Behavior \& Development, 29(3), 386-401. doi:10.1016/j. infbeh.2006.01.004

Rothbart, M. K. (2004). Commentary: Differentiated measures of temperament and multiple pathways to childhood disorders. Journal of Clinical Child and Adolescence Psychology, 33(1), 82-87. doi:10.1207/S15374424JCCP3301_8

Rothbart, M. K., \& Bates, J. E. (2006). Temperament. In W. Damon, R. M. Lerner, \& N. Eisenberg (Eds.), Handbook of child psychology: Social, emotional and personality development (Vol. 3, 6th ed, pp. 99-165), New York: John Wiley and Sons.

Sameroff, A. J. (2009). Conceptual issues in studying the development of self-regulation. In S. L. Olson \& A. J. Sameroff (Eds.), Biopsychosocial regulatory processes in the development of childhood behavioral problems (pp. 1-18). New York: Cambridge University Press.

Spitzer, R. L., Williams, J. B., Gibbon, M., \& First, M. B. (1989). Instruction manual for the structured clinical interview for DSM III - R (SCID, 5/11/89 Revision). New York: Biometrics Research Department.

Swanson, E. C., \& Schleiss, M. R. (2013). Congenital cytomegalovirus infection: New prospects for prevention and therapy. Pediatric Clinics of North America, 60(2), 335-349. doi:10.1016/j.pcl.2012.12.008

Taylor, Z. E., Eisenberg, N., Spinrad, T. L., \& Widaman, K. F. (2013). Longitudinal relations of intrusive parenting and effortful control to ego-resiliency during early childhood. Child Development, 84(4), 1145-1151. doi:10.1111/cdev.12054

Tomasello, M. (2003). Origens culturais da aquisição do conhecimento humano: Tópicos (C. Berliner, Trad.). São Paulo, SP: Martins Fontes.

Vygotsky, L. S. (1998). A formação social da mente (6. ed.). São Paulo, SP: Martins Fontes. (Original publicado em 1930) 